\title{
Some remarks on the selection of exit channels in the theoretical description of dissociative recombination
}

\author{
Steven L. Guberman ${ }^{\mathrm{a}}$ \\ Institute for Scientific Research, 22 Bonad Road, Winchester, MA 01890, USA
}

\begin{abstract}
The need for the careful selection of dissociative routes for inclusion in the theoretical calculation of accurate dissociative recombination (DR) cross sections and rate constants is discussed. For total energies corresponding to the sum of the "free" electron energy plus the ion vibrational energy, all neutral diabatic curves that fall between or near the turning points of the highest Rydberg vibrational levels with the same electronic symmetry need to be included in the calculation. This is especially the case if there are several dissociative routes of the same electronic symmetry that satisfy this criterion. Also, care must be exercised in the exclusion of dissociative routes having relatively small electron capture widths. Examples from theoretical studies of the dissociative recombination (DR) of $\mathrm{N}_{2}^{+}$are used as illustrations.
\end{abstract}

\section{Introduction}

In dissociative recombination (DR), after electron $\left(\mathrm{e}^{-}\right)$capture, the products separate on neutral repulsive states. For example, the DR of $\mathrm{N}_{2}^{+}$, the topic of this paper, is described by

$$
\mathrm{N}_{2}^{+}+\mathrm{e}^{-} \rightarrow \mathrm{N}+\mathrm{N} \text {. }
$$

In most diatomics, a plethora of exit channels determines the motion of the $\mathrm{N}$ atoms on the right hand side of (1). The huge number of possibilities can make theoretical dissociative recombination (DR) calculations appear to be formidable. For the atmospheric ions $\mathrm{O}_{2}^{+}, \mathrm{N}_{2}^{+}$and $\mathrm{NO}^{+}$, there are 61,50 and 27 energetically allowed states for DR from the lowest ion vibrational level at zero "free" electron energy. The quotes are used because the electron on the left side of (1) is not free but moves in the coulomb field of the ion. Of course, if one includes excited ion vibrational levels, the numbers are even higher. So how does one select the important routes? Some routes can be eliminated by spin and electronic reflection symmetry. For the remaining routes, a common approach has been to include all diabatic dissociative states that cross the ion potential curve within or near the turning points of the vibrational level undergoing DR. In this paper, the case is made that this approach will miss important dissociative states that contribute to cross section structure. Instead, due to the important role played by indirect recombination [1], the selection of dissociative states should be based upon whether they fall within or near the turning points of the highest Rydberg vibrational levels falling within the electron energy range

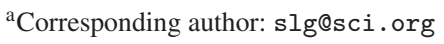

This is an Open Access article distributed under the terms of the Creative Commons Attribution License 4.0, which permits unrestricted use, distribution, and reproduction in any medium, provided the original work is properly cited. 


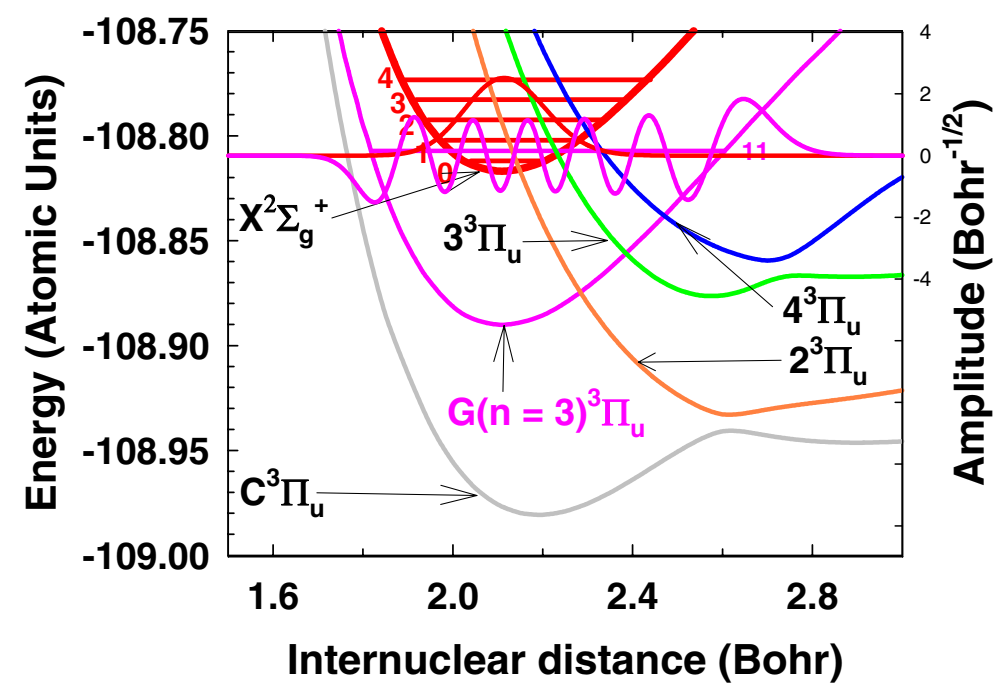

Figure 1. $C^{3} \Pi_{u}$ (gray online), $2^{3} \Pi_{u}$ (orange online), $3^{3} \Pi_{u}$ (green online) and $4^{3} \Pi_{u}$ (blue online) diabatic potential curves of $\mathrm{N}_{2}$ with the $\mathrm{v}=11$ vibrational level of the $\mathrm{G}^{3} \Pi_{\mathrm{u}}$ Rydberg state (pink online). Also shown is the ground state of $\mathrm{N}_{2}^{+}$(red online) with the $\mathrm{v}=0$ (red online) and $\mathrm{G}^{3} \Pi_{\mathrm{u}} \mathrm{v}=11$ (pink online) vibrational wave functions. The amplitude scale for the vibrational wave functions is on the right.

of interest. This approach will increase the number of relevant dissociative states compared to that which uses the ion turning points as the criterion. Also an example will be given which demonstrates the need for care when eliminating dissociative states simply because they have small electron capture widths.

In the next section, an example is given to demonstrate the selection issues relevant to the position of the dissociative curve. Section 3 has a discussion of the importance of a state having a very small electron capture width. The discussions are based upon recent studies of the $\mathrm{DR}$ of $\mathrm{N}_{2}^{+}[2,3]$. The conclusions are in Sect. 4 followed by an Acknowledgment in Sect. 5.

\section{Selection of dissociative states based upon position}

The reader is referred to earlier publications for the details of the ab initio calculation of the diabatic neutral, ion and diabatic Rydberg states [2,3]. In the direct recombination process [4], two quantities determine the relative importance of a dissociative route: the Franck-Condon (FC) factors between the dissociative and ion vibrational wave functions and the electron capture width. If the width is too small, the electron cannot be captured and if it is too large, the electron can be emitted before dissociation is complete. Below, I show that consideration of indirect recombination requires a revision of these rules.

Figure 1 shows the most important of the 14 dissociative states needed for the description of the DR of $\mathrm{N}_{2}^{+}$, the four ${ }^{3} \Pi_{\mathrm{u}}$ states. The overall importance was determined in the prior calculations [2, 3]. For DR from $\mathrm{v}=0,2^{3} \Pi_{\mathrm{u}}$ is quite important for direct DR since it crosses the level near the vibrational wave function maximum (see Fig. 1). In addition, it has the largest electron capture widths with the ground ion core of all the dissociative states (with the exceptions of $\mathrm{b}^{\prime 1} \Sigma_{\mathrm{u}}^{+}$and $2^{3} \Sigma_{\mathrm{u}}^{+}$). At $\mathrm{R}=2.1 \mathrm{a}_{\mathrm{o}}$, the width is $0.16 \mathrm{eV}$ [2]. This width is not large enough to make autoionization a major process. At first glance, the $\mathrm{C}$ state would not be expected to play an important role because it has a vanishingly small Franck-Condon (FC) factor with $\mathrm{v}=0$. Figure 1 shows that the $3^{3} \Pi_{\mathrm{u}}$ state has a FC factor that is likely to be nonnegligible. However, its electron capture width near $\mathrm{R}=2.1 \mathrm{a}_{\mathrm{o}}$ is a factor of 70 [2] smaller than that for $2^{3} \Pi_{\mathrm{u}}$ and one might assume that it will not play an important role for the DR of any of the ion vibrational levels [2]. The $4^{3} \Pi_{\mathrm{u}}$ state is expected to have only a small FC factor with $\mathrm{v}=0$ but 


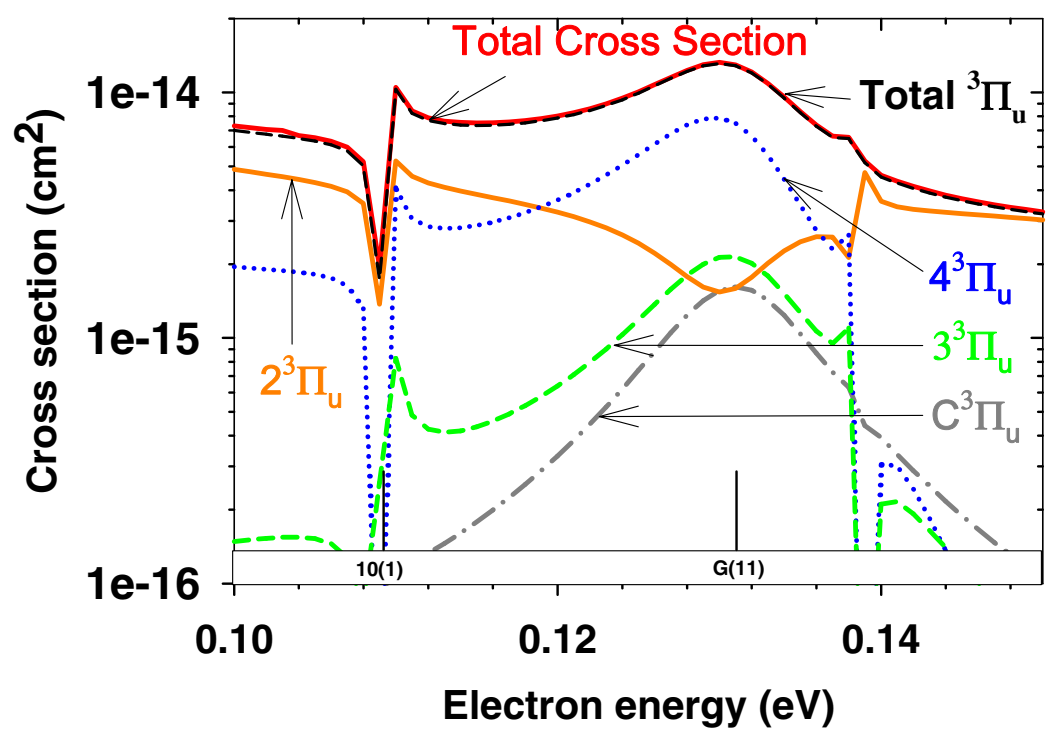

Figure 2. Cross section for $D R$ from $v=0$ in the electron energy range $0.10-0.15 \mathrm{eV}$. The state contributions to the cross section are shown for $C^{3} \Pi_{u}$ (gray online, dot-dashed), $2^{3} \Pi_{u}$ (orange online, solid), $3^{3} \Pi_{u}$ (green online, dashed) and $4^{3} \Pi_{u}$ (blue online, dotted). The total cross section is shown (solid, red online) as is the total for the ${ }^{3} \Pi_{u}$ states (black dashed).

possibly a higher factor with excited vibrational levels. The width is about a factor of four lower than that for $2^{3} \Pi_{u}[2]$.

In the Multichannel Quantum Defect Theory (MQDT) approach [5] used here, the dissociative routes can mix with each other because they are all connected to the Rydberg states by the predissociation electronic coupling. Insight into the role of indirect DR can be gained if we first ask: what is the cross section for DR along the $\mathrm{C}$ state if no other dissociative state is allowed to enter the calculation? As expected, the cross section from $\mathrm{v}=0$ is very low, i.e. less than $2 \times 10^{-17} \mathrm{~cm}^{2}$ over the electron energy range from 0.10 to $0.15 \mathrm{eV}$, the energy range of Fig. 2. If all dissociative states, including the four ${ }^{3} \Pi_{u}$ states, are allowed in the calculation, the cross sections shown in Fig. 2 are obtained [2]. Remarkably, at $0.13 \mathrm{eV}$, the contribution of $C^{3} \Pi_{u}$ to the total cross section is equal to that for $2^{3} \Pi_{u}$ and $4^{3} \Pi_{u}$ is the major component. What is the source of the unexpected magnitudes of these contributions?

The predissociation widths of the ${ }^{3} \Pi_{u}$ Rydberg vibrational levels for each of the dissociative ${ }^{3} \Pi_{u}$ states are present in the calculation [2]. These widths connect the dissociative states to each other. This connection is illustrated in Fig. 3 which also shows the $v=11$ level of $G^{3} \Pi_{u}$. This state is the lowest ${ }^{3} \Pi_{\mathrm{u}}$ Rydberg state having the ground $\mathrm{N}_{2}^{+}$core. From the quantum defects alone [2], this state is expected to be centered at $0.089 \mathrm{eV}$ above the ion $\mathrm{v}=0$. However, interaction with the dissociative continua causes a shift to higher energy of about $0.041 \mathrm{eV}$. Near $0.13 \mathrm{eV}$, the most probable dissociative state for electron capture is $2^{3} \Pi_{u}$. Capture into $2^{3} \Pi_{u}$ leads to a complex scenario in which all the paths interfere with each other in the calculations. Among these are autoionization of $2^{3} \Pi_{u}$, and excitation of the $v=11$ level of $G^{3} \Pi_{u}$ via $2^{3} \Pi_{u}$ followed by dissociation along all the ${ }^{3} \Pi_{u}$ valence states. Because the $\mathrm{G}$ state is connected via predissociation widths to all the dissociative states, capture into $2^{3} \Pi_{\mathrm{u}}$ leads to population of the $G$ state which serves as a feeder to each of the three other ${ }^{3} \Pi_{u}$ states. Each of the three other ${ }^{3} \Pi_{\mathrm{u}}$ states has a small direct recombination cross section but from the feeder state, they each contribute a peak to the cross section. Indeed, it is common in DR for dissociative paths that have weak direct cross sections to have peaks due to Rydberg resonances. These peaks are Lorentzian-like and are similar to those that would arise from the Breit-Wigner cross section expression [1] where interference 


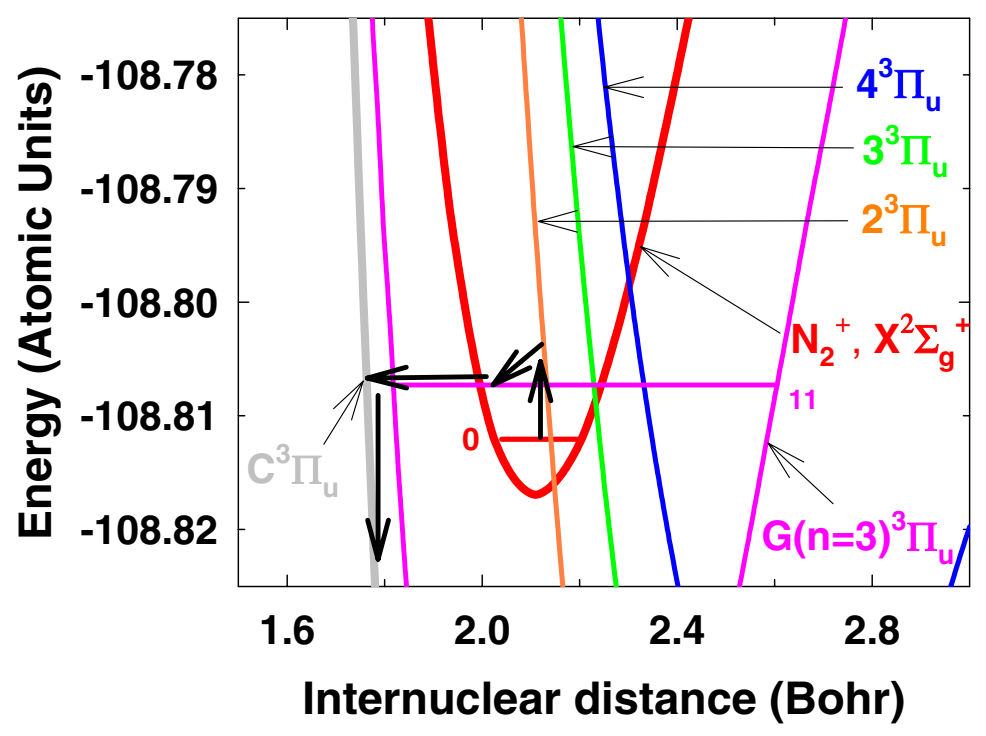

Figure 3. An enlargement of a portion of Fig. 1 is shown with added arrows showing the mechanism by which $\mathrm{G}^{3} \Pi_{\mathrm{u}}$ is populated.

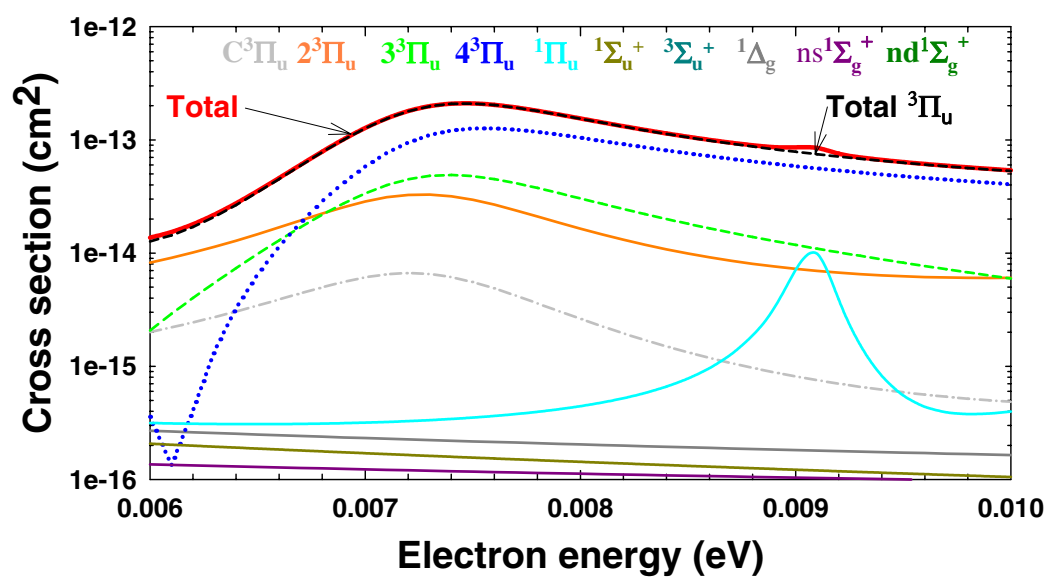

Figure 4. Total (solid, red) and partial cross sections from $v=1$ with the total cross section for ${ }^{3} \Pi_{u}($ dashed, black) [3]. The $3^{3} \Pi_{u}$ contribution is dashed and green.

with the background continua (due to direct DR) is ignored. (The Breit-Wigner expression is not used in these calculations). The peak occurs in the $3^{3} \Pi_{u}$ contribution because direct DR along $3^{3} \Pi_{u}$ has a small cross section due to the small electron capture width. Direct capture into $4^{3} \Pi_{u}$ is also small (due to its small FC factor) with the ion $\mathrm{v}=0$. However, a combination of the magnitude of its width and its $\mathrm{FC}$ factor with $\mathrm{v}=11$ makes it the dominant cross section contributor near $0.13 \mathrm{eV}$. For $2^{3} \Pi_{\mathrm{u}}$, direct recombination is strong and destructive interference with indirect recombination through $\mathrm{v}=11$ causes a dip in its cross section contribution. Lastly, autoionization of $\mathrm{v}=11$ to the $\mathrm{v}=0$ ground ion level is unlikely due to the large $\Delta \mathrm{v}$. 


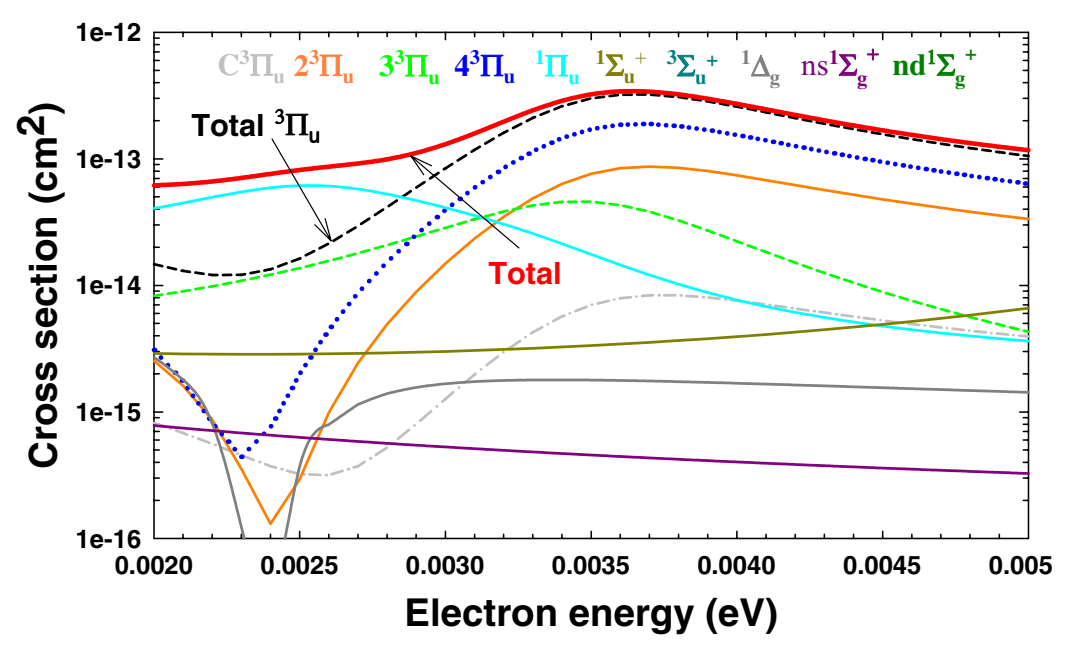

Figure 5. Total (solid, red) and partial cross sections from $v=2$ with the total cross section for ${ }^{3} \Pi_{u}$ (dashed, black) [3]. The $3^{3} \Pi_{u}$ contribution is dashed and green.

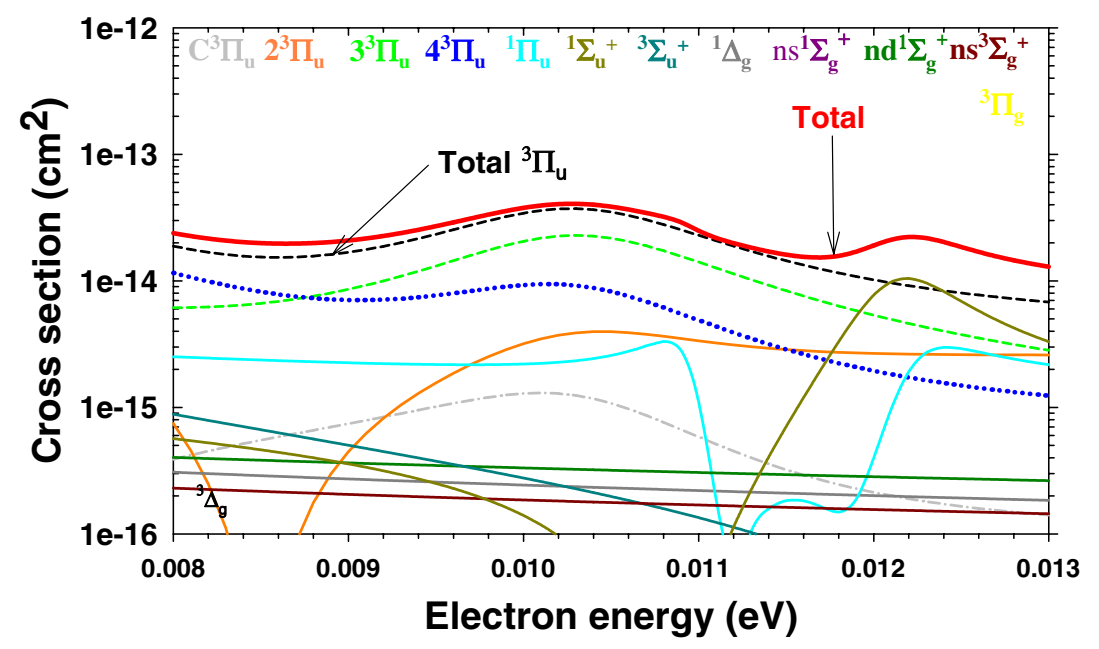

Figure 6. Total (solid, red) and partial cross sections from $v=3$ with the total cross section for ${ }^{3} \Pi_{u}$ (dashed, black) [3]. The $3^{3} \Pi_{u}$ contribution is dashed and green.

It should be noted that at the energy of the $\mathrm{v}=11$ level, the $\mathrm{C}$ state is about $0.05 \mathrm{a}_{\mathrm{o}}$ to smaller $\mathrm{R}$ from the $\mathrm{v}=11$ inner turning point. Therefore, in determining which electronic symmetries to include, one must also consider dissociative routes that are just outside of the Rydberg level turning points.

\section{Selection of dissociative states based upon width magnitudes}

The small electron capture width of $3^{3} \Pi_{u}$ has been mentioned above. However, the importance of a dissociative state is dependent not just upon its electron capture width but also upon the FC factor with ion and Rydberg vibrational levels. This is demonstrated below with examples of the $3^{3} \Pi_{u}$ contribution to the cross sections at isolated energies [3]. Figures 4-6 show unexpected cross section contributions 
to the $\mathrm{v}=1,2$ and 3 cross sections, respectively. Figure 4 shows that the DR cross section along $3^{3} \Pi_{\mathrm{u}}$ exceeds that along $2^{3} \Pi_{\mathrm{u}}$ between 0.0067 and $0.0099 \mathrm{eV}$ even though its electronic width is 70 times smaller than that for $2^{3} \Pi_{u}$. In this energy range, $3^{3} \Pi_{u}$ is the second most important dissociative route after $4^{3} \Pi_{\mathrm{u}}$. Figure 5 shows the $v=2$ cross section at electron energies between 0.0020 and $0.0050 \mathrm{eV}$. Between 0.0020 and $0.0029 \mathrm{eV}$, the $3^{3} \Pi_{\mathrm{u}}$ cross section exceeds all the other ${ }^{3} \Pi_{\mathrm{u}}$ contributions. At higher energies, 2 and $4^{3} \Pi_{u}$ are more important. For $v=3$, Fig. 6 shows the dominance of $3^{3} \Pi_{u}$ between 0.00877 and $0.0119 \mathrm{eV}$ electron energies. $3^{3} \Pi_{\mathrm{u}}$ dominates all the other $3^{3} \Pi_{\mathrm{u}}$ states up to $0.0130 \mathrm{eV}$. Above $0.0119 \mathrm{eV}, 2^{1} \Sigma_{\mathrm{g}}^{1}$ becomes the dominant route.

Clearly, the results show that the $3^{3} \Pi_{u}$ cross section contribution cannot be neglected even though its width is much smaller than those for 2 and $4^{3} \Pi_{u}$. FC factors plus the coupling to the other dissociative states of the same electronic symmetry (see prior section) compensate for the small width allowing $3^{3} \Pi_{u}$ to dominate in narrow energy regions.

\section{Conclusions}

What are the implications of the current study for the calculation of DR cross sections for other molecular ions? I have shown that that a repulsive curve $\left(\mathrm{C}^{3} \Pi_{\mathrm{u}}\right)$ which at the energy of the $\mathrm{v}=0$ ion level is about $0.3 \mathrm{a}_{\mathrm{o}}$ to smaller $\mathrm{R}$ from the inner turning point of that level cannot be neglected if one aims for an accurate $\mathrm{v}=0 \mathrm{DR}$ cross section. This is especially true in cases where there are other repulsive states of the same electronic symmetry that can act as feeder states (through an intermediate Rydberg level) to a state that cannot be initially populated by the direct mechanism. In such cases, a peak can be expected in the cross section contribution from the distant states. Another possibility (not discussed in this paper) in cases where there is a rapid variation of the quantum defect with $\mathrm{R}$ is capture into a Rydberg level by Born-Oppenheimer breakdown followed by a distribution of the dissociating flux among repulsive states that cross the Rydberg level within or near its turning points.

I have also shown that if one is calculating DR cross sections for several vibrational levels and one dissociative route has an electronic width that is a factor of seventy lower than the most important state, it would not be wise to eliminate the smaller width state from the calculation. For molecules in which more than one dissociative state of the same electronic symmetry is present, the dissociative states can be connected through the Rydberg states and repulsive routes with small widths can be important in narrow energy regions.

This material is based upon work supported by the National Science Foundation under Grant No. 0838061.

\section{References}

[1] J. N. Bardsley, J. Phys. B 1, 365 (1968)

[2] S. L. Guberman, J. Chem. Phys. 137, 074309 (2012)

[3] S. L. Guberman, J. Chem. Phys. 139, 124318 (2013)

[4] D. R. Bates, Phys. Rev. 78, 492 (1950)

[5] S. L. Guberman and A. Giusti-Suzor, J. Chem. Phys. 95, 2602 (1991) 